\title{
Glucose 6-Phosphate Dehydrogenase and 6-Phosphogluconate Dehydrogenase Activities and Glucose Utilization by Species within the Genera Bacteroides, Prevotella, and Porphyromonas
}

\author{
GRAHAM D. BAILEY* AND DARIA N. LOVE \\ Department of Veterinary Pathology, University of Sydney, Sydney, New South Wales 2006, Australia
}

\begin{abstract}
Members of the genera Bacteroides, Prevotella, and Porphyromonas were investigated for their glucose 6-phosphate dehydrogenase (G6PDH) and 6-phosphogluconate dehydrogenase (6PGDH) activities by using spectrophotometric (SP) and alloenzyme elecrophoresis (AE) detection methods. When the SP and AE methods were compared, the AE method failed to detect activity in two of the six strains which exhibited G6PDH and 6PGDH activities as determined by the SP detection method. On the basis of the results of SP detection, Bacteroides levii ATCC $29147^{\mathrm{T}}$ ( $\mathrm{T}=$ type strain) (G6PDH and 6PGDH negative) is not a member of the genus Bacteroides as currently defined, which reflects recent 16S rRNA placement, nor do Prevotella heparinolytica ATCC 35895 , Prevotella zoogleoformans ATCC $33285^{\mathrm{T}}$, Porphyromonas canoris $12835^{\mathrm{T}}$, and Porphyromonas salivosa NCTC $11632^{\mathrm{T}}$ (all G6PDH and 6PGDH positive) conform to their respective genus descriptions. When these organisms were grown in prereduced peptone-yeast extract broth containing $10 \%(\mathrm{wt} / \mathrm{vol})$ glucose, the amounts of glucose remaining after 5 days were less than the amounts present initially for members of the genus Bacteroides (Bacteroides fragilis ATCC $25285^{\mathrm{T}}$ and $B$. levii) and members of the genus Prevotella (Prevotella melaninogenica ATCC $25845^{\mathrm{T}}$, Prevotella buccae ATCC $33574^{\mathrm{T}}$, Prevotella heparinolytica, and Prevotella zoogleoformans). In addition, the glucose levels were lower after 5 days of incubation in broth media containing Porphyromonas asaccharolytica ATCC $25845^{\mathrm{T}}$ and Porphyromonas salivosa, but not in media containing the other members of the genus Porphyromonas tested (Porphyromonas canoris, Porphyromonas circumdentaria NCTC $12469^{\mathrm{T}}$, Porphyromonas endodontalis ATCC 35406 ${ }^{\mathrm{T}}$, and Porphyromonas gingivalis ATCC $33277^{\mathrm{T}}$ ). The reductions in glucose levels were not directly related to the final $\mathrm{pH}$ values. Our results suggest that the descriptions of the genera Bacteroides, Prevotella, and Porphyromonas may require emendation to reflect variability in G6PDH and 6PGDH activities and glucose utilization.
\end{abstract}

The genus Bacteroides as described in Bergey's Manual of Systematic Bacteriology (5) contains a heterogeneous collection of species (11). This has led to nomenclature changes, including the establishment of the genus Porphyromonas for the "asaccharolytic pigmented Bacteroides spp." (12) and the establishment of the genus Prevotella for the "moderately saccharolytic, predominately oral Bacteroides spp." (14). In addition, the description of the genus Bacteroides has been emended so that this genus contains "only the saccharolytic, bile tolerant strains" (13). Since these changes, workers have published 16S rRNA sequence data which have shown that some species currently classified in the genera Bacteroides and Prevotella do not cluster with other members of these genera (10). In the descriptions of the redefined genera, the methods used to determine whether organisms were saccharolytic, moderately saccharolytic, or asaccharolytic were not specified. However, the descriptions included references to studies in which workers used acidification or failure to acidify media containing carbohydrates and/or changes in glucose concentrations as the determinants for defining organisms as saccharolytic or asaccharolytic $(5,17)$. A literal definition of a saccharolytic organism is an organism that is capable of breaking down carbohydrate(s). Acids may be produced from substances other than carbohydrates (e.g., amino acids, purines, pyrimidines), and metabolites (e.g., ammonia) may affect the final $\mathrm{pH}$ values of media. Therefore, a lower terminal $\mathrm{pH}$ does not necessarily indicate that carbohydrates are broken down and cannot be used alone as an indicator of saccharolytic activity.

\footnotetext{
* Corresponding author
}

One of the components of the emended descriptions of the genera Bacteroides, Prevotella, and Porphyromonas was whether glucose 6-phosphate dehydrogenase (G6PDH) and 6-phosphogluconate dehydrogenase (6PGDH) activities were present (12-14). The inclusion of these dehydrogenase activity characteristics was based on studies $(11,16)$ in which workers used a spectrophotometric (SP) method to detect dehydrogenase activity and alloenzyme electrophoresis (AE) to determine the electrophoretic mobilities in strains that exhibited dehydrogenase enzyme activity when the SP technique was used. These workers concluded that members of the genus Bacteroides exhibit both G6PDH and 6PGDH activities, while members of the genera Porphyromonas and Prevotella did not exhibit either enzyme activity at detectable levels. However, valid descriptions of some Prevotella and Porphyromonas species either have not included dehydrogenase activity data $(1,9,20)$ or have included dehydrogenase activity data based only on AE results $(6,15)$. However, there apparently have been no reports of comparisons of the SP and AE detection methods, and thus it is not known if the two techniques give identical results. In this paper we describe a comparison of Bacteroides, Prevotella, and Porphyromonas species in which the SP and AE techniques were used to determine G6PDH and 6PGDH activities. We also examined the association between the final $\mathrm{pH}$ values of glucose-based media and the ability of selected Bacteroides, Prevotella, and Porphyromonas species to break down glucose.

\section{MATERIALS AND METHODS}

Bacterial strains. The strains which we used were Bacteroides fragilis ATCC $25285^{\mathrm{T}}\left(\mathrm{T}=\right.$ type strain) and Bacteroides levii ATCC $29147^{\mathrm{T}}$, which were obtained from the American Type Culture Collection; Prevotella melaninogenica VPI $2381^{\mathrm{T}}$ (= ATCC $25845^{\mathrm{T}}$ ), which was obtained from L. V. H. Moore; 
TABLE 1. G6PDH and 6PGDH activities of selected Bacteroides, Prevotella, and Porphyromonas strains as determined by the $\mathrm{SP}$ and $\mathrm{AE}$ methods ${ }^{a}$

\begin{tabular}{|c|c|c|c|c|}
\hline \multirow{3}{*}{ Strain $^{b}$} & \multicolumn{4}{|c|}{ Enzyme activities } \\
\hline & \multicolumn{2}{|c|}{ SP method (nmol mg of protein ${ }^{-1} \min ^{-1}$ ) } & \multicolumn{2}{|c|}{ AE method ${ }^{c}$} \\
\hline & G6PDH & $6 \mathrm{PGDH}$ & G6PDH & $6 \mathrm{PGDH}$ \\
\hline Bacteroides fragilis ATCC $25285^{\mathrm{T}}$ & 96 & 36 & + & + \\
\hline Prevotella heparinolytica ATCC $35895^{\mathrm{T}}$ & 20 & 18 & - & - \\
\hline Prevotella zoogleoformans ATCC $33285^{\mathrm{T}}$ & 30 & 6 & $\mathrm{ND}^{d}$ & ND \\
\hline Porphyromonas canoris NCTC $12835^{\mathrm{T}}$ & 66 & 63 & + & + \\
\hline Porphyromonas canoris VPB 4879 & 58 & 38 & + & + \\
\hline Porphyromonas canoris VPB 4882 & 41 & 62 & + & + \\
\hline Porphyromonas salivosa NCTC $11632^{\mathrm{T}}$ & 48 & 14 & - & - \\
\hline
\end{tabular}

${ }^{a}$ When we used the SP method, we did not detect G6PDH and 6PGDH activities in B. levii ATCC $29147^{\mathrm{T}}$, Prevotella melaninogenica ATCC $25845^{\mathrm{T}}$, Prevotella bivia ATCC $29303^{\mathrm{T}}$, Prevotella buccae ATCC $33574^{\mathrm{T}}$, Prevotella buccalis ATCC $35310^{\mathrm{T}}$, Prevotella corporis ATCC $33547^{\mathrm{T}}$, Prevotella disiens ATCC $29426^{\mathrm{T}}$, Prevotella intermedia ATCC $25611^{\mathrm{T}}$, Prevotella loescheii ATCC $15930^{\mathrm{T}}$, Prevotella oralis ATCC $33269^{\mathrm{T}}$, Prevotella oris ATCC $33573^{\mathrm{T}}$, Prevotella veroralis ATCC $33779^{\mathrm{T}}$, Porphyromonas asaccharolytica ATCC $25260^{\mathrm{T}}$, and Porphyromonas endodontalis ATCC $35406^{\mathrm{T}}$. G6PDH and 6PGDH activities were not detected in Porphyromonas circumdentaria NCTC $12469^{\mathrm{T}}$ and Porphyromonas gingivalis ATCC $33277^{\mathrm{T}}$, VPB 3318, and VPB 3492 by the SP and AE methods.

${ }^{b}$ ATCC, American Type Culture Collection; NCTC, National Collection of Type Cultures; VPB, Veterinary Pathology and Bacteriology.

${ }^{c}+$, enzyme activity band detected; -, enzyme activity band not detected.

${ }^{d} \mathrm{ND}$, not done.

Prevotella zoogleoformans VPI D28K-1 ${ }^{\mathrm{T}}$ (= ATCC $33285^{\mathrm{T}}$ ), which was obtained from the late E. P. Cato; Prevotella bivia ATCC $29303^{\mathrm{T}}$, Prevotella corporis ATCC $33547^{\mathrm{T}}$, Prevotella disiens ATCC $29426^{\mathrm{T}}$, Prevotella intermedia ATCC $25611^{\mathrm{T}}$ and Prevotella heparinolytica ATCC $35895^{\mathrm{T}}$, which were obtained from the American Type Culture Collection; Prevotella buccae CCUG $15401^{\mathrm{T}}$ (= ATCC $33574^{\mathrm{T}}$ ), Prevotella buccalis CCUG $15557^{\mathrm{T}}$ (= ATCC $\left.35310^{\mathrm{T}}\right)$, Prevotella loescheii CCUG $5914^{\mathrm{T}}\left(=\right.$ ATCC $15930^{\mathrm{T}}$ ), Prevotella oralis CCUG $15408^{\mathrm{T}}$ (= ATCC $33269^{\mathrm{T}}$ ), Prevotella oris CCUG $15405^{\mathrm{T}}\left(=\right.$ ATCC $33573^{\mathrm{T}}$ ), and Prevotella veroralis CCUG $15422^{\mathrm{T}}$ (= ATCC $33779^{\mathrm{T}}$ ), which were obtained from the Culture Collection of the University of Göteborg; Porphyromonas asaccharolytica ATCC $25268^{\mathrm{T}}$ and Porphyromonas gingivalis ATCC $33277^{\mathrm{T}}$, which were obtained from the American Type Culture Collection; and Porphyromonas salivosa VPB $157^{\mathrm{T}}$ (= NCTC $\left.11632^{\mathrm{T}}\right)$, Porphyromonas circumdentaria VPB $3329^{\mathrm{T}}$ (= NCTC $12469^{\mathrm{T}}$ ), feline strains Porphyromonas gingivalis VPB 3318 and VPB 3492 (7), Porphyromonas canoris VPB $4878^{\mathrm{T}}$ (= NCTC $12835^{\mathrm{T}}$ ), Porphyromonas canoris VPB 4879 and VPB 4882 (8), and Porphyromonas endodontalis CCUG $16442^{\mathrm{T}}$ $\left(=\right.$ ATCC $\left.35406^{\mathrm{T}}\right)$, which were obtained from the Culture Collection of the University of Göteborg.

Preparation of bacterial extracts. The general methods used for growth were the methods described by Holdeman et al. (4). Cells were harvested following 16 $h$ of growth in prereduced brain heart infusion broth (4) supplemented with $4 \mathrm{ml}$ of salts solution (4) per liter and $0.47 \%$ (wt/vol) glucose or following 2 to 3 days of growth on sheep blood agar supplemented with hemin-menadione, formate fumarate, and Proteose Peptone (6). The cells were washed twice with cold $\left(5^{\circ} \mathrm{C}\right)$ distilled water and stored at $-140^{\circ} \mathrm{C}$. The cells were lysed by sonication at $4^{\circ} \mathrm{C}$ with a model 375 sonicator (Heat Systems Ultrasonics, Inc.) (cup horn power output, 6 ) for $6 \mathrm{~min}$ or by passing them twice through a cold French pressure cell (Aminco model Fa-030) at $110 \mathrm{MPa}$. Following lysis, preparations were centrifuged at $17,000 \times g$ for $20 \mathrm{~min}$ in a refrigerated $\left(5^{\circ} \mathrm{C}\right)$ centrifuge to remove cellular debris.

SP determination of G6PDH and 6PGDH activities. We used the SP method of Deutsch (3), with some modifications. Briefly, G6PDH activity was measured at $340 \mathrm{~nm}$ with maleimide (an inhibitor of $6 \mathrm{PGDH}$ ) in the reaction mixture by using a Gilford model 260 spectrophotometer at $37^{\circ} \mathrm{C}$. Negative controls in which distilled water was substituted for substrate were included. The 6PGDH assay was performed like the G6PDH assay except that maleimide was omitted from the assay mixture and 6-phosphogluconate was used as the substrate. The activity values reported below were the averages of the values from three separate assays per preparation and were determined by using an extinction coefficient for NADPH of $6.22 \times 10^{2}$. The Bio-Rad protein assay (Bio-Rad, Richmond, Calif.) was used as recommended by the manufacturer to determine the protein concentration of each preparation; bovine serum albumin was used as the protein standard.

AE. AE was performed with selected isolates as described previously (6) Extracts were applied to gels by placing approximately $1 \mu \mathrm{l}$ of bacterial extract containing 6 to $7 \mathrm{mg}$ of protein per $\mathrm{ml}$ on a gel as a single line. Gels were read at $15 \mathrm{~min}$ to determine enzyme activity band formation.

Utilization of glucose and $\mathrm{pH}$ values of glucose-containing media (final pH values and changes in $\mathbf{p H}$ values). Glucose utilization was determined for the type species of the genera Bacteroides, Prevotella, and Porphyromonas and for selected other species belonging to these genera (see Table 2). For each strain, three single colonies were inoculated into cooked meat medium (bovine viandefoie broth [19] containing chopped meat, cysteine, and hemin-menadione [4]) and grown for 18 to $24 \mathrm{~h}$ at $37^{\circ} \mathrm{C}$. Each broth preparation was checked for purity by Gram staining and was inoculated into two tubes containing prereduced peptone-yeast extract-glucose broth (PYG) (4). Immediately following inoculation, each tube was vortexed for $20 \mathrm{~s}$. We let each tube stand for $20 \mathrm{~s}$, and then a $3-\mathrm{ml}$ aliquot was removed. The $\mathrm{pH}$ of the aliquot was determined (this was the initial $\mathrm{pH})$, the aliquot was centrifuged at $12,000 \times \mathrm{g}$ for $20 \mathrm{~min}$, and the supernatant was removed. This supernatant was mixed by vortexing, and three $100-\mu l$ aliquots were removed, mixed with equal volumes of distilled water, and then stored at $-20^{\circ} \mathrm{C}$ until they were assayed for their glucose contents. The inoculated tubes were incubated at $37^{\circ} \mathrm{C}$ for 7 days, Gram stained, and subcultured aerobically to check for purity before the frozen aliquots were assayed. A negative control consisted of PYG inoculated with sterile cooked meat medium and processed as described above. Glucose levels were determined with a Cobas Mira instrument (Hoffman-La Roche Diagnostics, Basle, Switzerland) by using an enzymatic glucose reagent (hexokinase) (Trace Scientific, Clayton, Australia) as described by the manufacturer.

\section{RESULTS}

The results of SP determinations of enzyme activities are presented in Table 1. B. fragilis exhibited G6PDH and 6PGDH activities of 96 and $36 \mathrm{nmol} \mathrm{mg}$ of protein ${ }^{-1} \mathrm{~min}^{-1}$, respectively, while 2 of 13 Prevotella spp. exhibited G6PDH activities of 20 and $30 \mathrm{nmol} \mathrm{mg} \mathrm{of} \mathrm{protein}{ }^{-1} \mathrm{~min}^{-1}$ and $6 \mathrm{PGDH}$ activities of 6 and $18 \mathrm{nmol} \mathrm{mg}$ of protein ${ }^{-1} \mathrm{~min}^{-1}$. Four of the 10 members of the genus Porphyromonas tested exhibited G6PDH activities which ranged from 41 to $66 \mathrm{nmol} \mathrm{mg}$ of protein ${ }^{-1} \mathrm{~min}^{-1}$ and $6 \mathrm{PGDH}$ activities which ranged from 14 to $63 \mathrm{nmol} \mathrm{mg} \mathrm{of} \mathrm{protein}{ }^{-1} \mathrm{~min}^{-1}$. G6PDH and 6PGDH activities were not detected in extracts of $B$. levii, 11 of the 13 Prevotella strains tested, and 6 of the 10 Porphyromonas strains tested.

Table 1 also shows the results of a comparison of the SP and $\mathrm{AE}$ methods for detecting G6PDH and 6PGDH activities. When the AE method was used, activity was not discernible until more than $0.27 \mathrm{U}$ of G6PDH (1 U was defined as the amount of enzyme which converted $1 \mu \mathrm{mol}$ of NADP to NADPH in $1 \mathrm{~min}$ ) or $0.11 \times 10^{-3} \mathrm{U}$ of $6 \mathrm{PGDH}$ was applied to the gel. When we increased the enzyme detection time (to more than $30 \mathrm{~min}$ ), we observed weak bands with Prevotella heparinolytica, Prevotella zoogleoformans, and Porphyromonas salivosa. However, in our hands this procedure increased the potential for development of staining artifacts which could be reported as false-positive reactions in strains for which no activity was detected when the SP method was used.

The results of tests for glucose utilization and $\mathrm{pH}$ following growth in PYG are shown in Table 2. We found that the 
TABLE 2. Utilization of glucose and final $\mathrm{pH}$ values following incubation of selected Bacteroides, Prevotella, and Porphyromonas strains in PYG

\begin{tabular}{|c|c|c|c|c|c|}
\hline \multirow[b]{2}{*}{ Strain $^{a}$} & \multicolumn{3}{|c|}{ Glucose in PYG } & \multirow{2}{*}{$\begin{array}{l}\text { Final pH } \\
\text { of PYG }\end{array}$} & \multirow{2}{*}{$\begin{array}{c}\mathrm{pH} \\
\text { change }\end{array}$} \\
\hline & $\begin{array}{c}\text { Initial concn } \\
\text { (nmol/liter) }\end{array}$ & $\begin{array}{l}\text { Final concn } \\
\text { (nmol/liter) }\end{array}$ & $\begin{array}{c}\% \\
\text { Reduction }\end{array}$ & & \\
\hline Bacteroides fragilis ATCC $25285^{\mathrm{T}}$ & 32 & 16 & 50 & 5.3 & 1.4 \\
\hline Bacteroides levii ATCC $29147^{\mathrm{T}}$ & 33 & 14 & 58 & 5.6 & 1.0 \\
\hline Prevotella melaninogenica ATCC $25845^{\mathrm{T}}$ & 28 & 7 & 75 & 4.8 & 1.9 \\
\hline Prevotella buccae ATCC $33574^{\mathrm{T}}$ & 31 & 6 & 81 & 4.6 & 2.1 \\
\hline Prevotella heparinolytica ATCC $35895^{\mathrm{T}}$ & 34 & 18 & 47 & 5.6 & 1.0 \\
\hline Prevotella zoogleoformans ATCC $33285^{\mathrm{T}}$ & 35 & 10 & 71 & 5.6 & 0.9 \\
\hline Porphyromonas asaccharolytica ATCC $25260^{\mathrm{T}}$ & 32 & 19 & 41 & 6.2 & 0.5 \\
\hline Porphyromonas circumdentaria NCTC $12469^{\mathrm{T}}$ & 32 & 33 & 0 & 6.3 & 0.3 \\
\hline Porphyromonas canoris NCTC $12835^{\mathrm{T}}$ & 32 & 32 & 0 & 6.6 & 0 \\
\hline Porphyromonas endodontalis ATCC $35406^{\mathrm{T}}$ & 29 & 32 & 0 & 6.6 & 0 \\
\hline Porphyromonas gingivalis ATCC $33277^{\mathrm{T}}$ & 31 & 37 & 0 & 6.6 & 0 \\
\hline Porphyromonas salivosa $\mathrm{NCTC} 11632^{\mathrm{T}}$ & 33 & 13 & 60 & 5.9 & 0.7 \\
\hline
\end{tabular}

${ }^{a}$ ATCC, American Type Culture Collection; NCTC, National Collection of Type Cultures.

amounts of glucose that remained after 5 days of growth were less than the amounts present initially for all Bacteroides spp. and Prevotella spp. tested. The glucose levels were also lower after 5 days of incubation in broth media containing Porphyromonas asaccharolytica (32 to $19 \mathrm{mmol} / \mathrm{liter}$ ) and Porphyromonas salivosa (33 to $13 \mathrm{mmol} /$ liter) but not in media containing the other Porphyromonas spp. tested.

Table 2 shows the final pH values in PYG. Incubation of $B$. fragilis for 5 days reduced the $\mathrm{pH}$ of the medium by $1.4 \mathrm{pH}$ units to a final $\mathrm{pH}$ of 5.3, and incubation of $B$. levii reduced the $\mathrm{pH}$ of the medium by $1.0 \mathrm{pH}$ unit to a final $\mathrm{pH}$ of 5.6. Members of the genus Prevotella likewise reduced the $\mathrm{pH}$ values of PYG by 0.9 to $2 \mathrm{pH}$ units, with final $\mathrm{pH}$ values ranging from 4.6 to 5.7. However, Porphyromonas strains either caused no change in the $\mathrm{pH}$ (final $\mathrm{pH}$ value range, 6.3 to 6.6 ) or produced changes of 0.5 and $0.7 \mathrm{pH}$ unit (final $\mathrm{pHs}, 6.2$ and 5.9, respectively).

\section{DISCUSSION}

Basic to the nomenclature of the anaerobic, gram-negative, rod-shaped bacteria have been descriptions in which the socalled saccharolytic activities and G6PDH and 6PGDH activities of strains have been used. Using the SP method to detect these enzyme activities, we obtained results that were similar to those of Shah and Williams (16) for the six strains common to our report and the report of Shah and Williams despite quantitative differences in G6PDH and 6PGDH enzyme activities. When the SP and AE methods were compared, however, the $\mathrm{AE}$ method failed to detect activity in two of the six strains which exhibited G6PDH and 6PGDH activities as determined by the SP method (Table 1). While some workers have reported G6PDH and 6PGDH activities on the basis of AE data, our results suggest that only the SP technique should be used to detect these activities until a simple, more reliable technique becomes available. On the basis of SP detection, $B$. levii (G6PDH and 6PGDH negative) is not a member of the genus Bacteroides as currently defined, nor do Prevotella heparinolytica, Prevotella zoogleoformans, Porphyromonas canoris, and Porphyromonas salivosa (all G6PDH and 6PGDH positive) conform completely to their respective genus descriptions (Table 1). This brings into question the usefulness of G6PDH and 6PGDH activities as key determinants of species identity in the genera Bacteroides, Prevotella, and Porphyromonas as currently defined. Alternately, placement of these species in their current genera is not valid.

Table 2 shows that the final $\mathrm{pH}$ was not necessarily a direct indication of saccharolytic activity. For example, $B$. fragilis, Prevotella buccae, Prevotella melaninogenica, Prevotella heparinolytica, Prevotella zoogleoformans, Porphyromonas asaccharolytica, and Porphyromonas salivosa cultures exhibited decreases in the levels of measurable glucose ranging from 41 to $81 \%$. While the decreases in the $\mathrm{pH}$ values of the media ranged from $0.5 \mathrm{pH}$ unit (Porphyromonas asaccharolytica) to $2.1 \mathrm{pH}$ units (Prevotella buccae), these decreases were not consistent with the reductions in measurable glucose levels, nor were they correlated with the current division into saccharolytic, moderately asaccharolytic, and asaccharolytic taxa. Given that the final $\mathrm{pH}$ is the result of a range of activities (utilization of carbohydrate is only one potential activity), these findings suggest that workers need to be careful when they interpret a change in the $\mathrm{pH}$ of peptone-carbohydrate medium as indicating that carbohydrate utilization has occurred. Our results illustrate that the current primary division of the genera Bacteroides, Prevotella, and Porphyromonas based on final $\mathrm{pH}$ values in carbohydrate media after incubation (as an indication of the ability to utilize carbohydrate) needs to be reconsidered.

The current classification of the genera Bacteroides, Prevotella, and Porphyromonas places emphasis on physiological characteristics (18). However, 16S rRNA sequence data have shown that Porphyromonas asaccharolytica, Porphyromonas canoris, Porphyromonas circumdentaria, Porphyromonas endodontalis, Porphyromonas gingivalis, Porphyromonas salivosa, and $B$. levii cluster together $(2,10)$. As three of these seven species are saccharolytic, it appears that the genus Porphyromonas should not be defined as consisting only of asaccharolytic organisms. Similarly, while the genus has been defined as consisting of species which fail to produce G6PDH and 6PGDH, Porphyromonas canoris and Porphyromonas salivosa produce both enzymes and thus do not conform completely to the current genus definition. These taxa are, however, deeply embedded in the Porphyromonas cluster $(2,10)$. A $16 \mathrm{~S}$ rRNA sequence analysis of Prevotella heparinolytica and Prevotella zoogleoformans has shown that these species are most closely related to each other and related (albeit distantly) to members of the genus Bacteroides $(2,10)$. Paster et al. (10) proposed that Prevotella heparinolytica and Prevotella zoogleoformans belong to the Bacteroides cluster, which is consistent with our dehy- 
drogenase and saccharolytic activity results. However, transfer of these species to the genus Bacteroides would require emendation of the current genus definition (both species fail to grow in the presence of bile and to date have been isolated only from oral cavities or individuals with oral diseases). It may be necessary to form a new genus to accommodate these species. 16S rRNA sequence data have revealed that the remaining Prevotella spp. which we studied (in which G6PDH and 6PGDH activities were not detected) grouped distinctly from the $\mathrm{Bac}$ teroides and Porphyromonas clusters but possessed some of the characteristics of both of these genera (i.e., they are saccharolytic but do not exhibit G6PDH and 6PGDH activities).

\section{ACKNOWLEDGMENTS}

Prereduced and other bacteriological media were prepared by $\mathrm{L}$. Patoka and F. Taeker. D. Griffin performed the glucose measurements. cil.

This work was supported in part by the Australian Research Coun-

\section{REFERENCES}

1. Cato, E. P., R. W. Kelley, W. E. C. Moore, and L. V. Holdeman. 1982. Bacteroides zoogleoformans (Weinberg, Nativelle, and Prevot 1937) corrig., comb. nov.: emended description. Int. J. Syst. Bacteriol. 32:271-274.

2. Collins, M. D., D. N. Love, J. Karjalainen, A. Kanervo, B. Forsblom, A. Willems, S. Stubbs, E. Sarkiala, G. D. Bailey, D. I. Wigney, and H. Jousimies-Somer. 1994. Phylogenetic analysis of members of the genus Porphyromonas and description of Porphyromonas cangingivalis sp. nov. and Porphyromonas cansulci sp. nov. Int. J. Syst. Bacteriol. 44:674-679.

3. Deutsch, J. 1983. Glucose-6-phosphate dehydrogenase, p. 190-197. In H. V. Bergmeyer (ed.), Methods in enzymatic analysis, vol. 3. Verlag Chemie, Weinheim, Germany.

4. Holdeman, L. V., E. P. Cato, and W. E. C. Moore. (ed). 1977. Anaerobe laboratory manual. Virginia Polytechnic Institute and State University, Blacksburg.

5. Holdeman, L. V., R. W. Kelley, and W. E. C. Moore. 1984. Family I. Bacteroidaceae Pribham 1983,10 AL , p. 602-662. In N. R. Krieg and J. G. Holt (ed.), Bergey's manual of systematic bacteriology, vol. 1. The Williams \& Wilkins Co., Baltimore.

6. Love, D. N., G. D. Bailey, S. Collings, and D. A. Briscoe. 1992. Description of Porphyromonas circumdentaria sp. nov. and reassignment of Bacteroides salivosus (Love, Johnson, Jones, and Calverley 1987) as Porphyromonas (Shah and Collins 1988) salivosa comb. nov. Int. J. Syst. Bacteriol. 42:434 438 .

7. Love, D. N., J. L. Johnston, R. F. Jones, and A. Calverley. 1987. Bacteroides salivosus sp. nov., an asaccharolytic, black-pigmented species from cats. Int. J. Syst. Bacteriol. 37:307-309.

8. Love, D. N., J. Karjalainen, A. Kanervo, B. Forsblom, E. Sarkiala, G. D. Bailey, D. I. Wigney, and H. Jousimies-Somer. 1994. Porphyromonas canoris sp. nov., an asaccharolytic, black-pigmented species from the gingival sulcus of dogs. Int. J. Syst. Bacteriol. 44:204-208.

9. Okuda, K., T. Kato, J. Shiozu, I. Takazoe, and T. Nakamura. 1985. Bacteroides heparinolyticus sp. nov. isolated from humans with periodontitis. Int. J. Syst. Bacteriol. 35:438-442.

10. Paster, B. J., F. E. Dewhirst, I. Olson, and G. J. Fraser. 1994. Phylogeny of Bacteroides, Prevotella, and Porphyromonas spp. and related bacteria. J. Bacteriol. 176:725-732.

11. Shah, H. N., and M. D. Collins. 1983. A review. Genus Bacteroides. A chemotaxonomical perspective. J. Appl. Bacteriol. 55:403-416.

12. Shah, H. N., and M. D. Collins. 1988. Proposal for reclassification of Bacteroides asaccharolyticus, Bacteroides gingivalis, and Bacteroides endodontalis in a new genus, Porphyromonas. Int. J. Syst. Bacteriol. 38:128-131.

13. Shah, H. N., and M. D. Collins. 1989. Proposal to restrict the genus Bacteroides (Castellani and Chalmers) to Bacteroides fragilis and closely related species. Int. J. Syst. Bacteriol. 39:85-87.

14. Shah, H. N., and M. D. Collins. 1990. Prevotella, a new genus to include Bacteroides melaninogenicus and related species formerly classified in the genus Bacteroides. Int. J. Syst. Bacteriol. 40:205-208.

15. Shah, H. N., and S. E. Gharbia. 1992. Biochemical and chemical studies on strains designated Prevotella intermedia and proposal of a new pigmented species, Prevotella nigrescens sp. nov. Int. J. Syst. Bacteriol. 42:542-546.

16. Shah, H. N., and R. A. D. Williams. 1982. Dehydrogenase patterns in the taxonomy of Bacteroides. J. Gen. Microbiol. 128:2955-2965.

17. Shah, H. N., and R. A. D. Williams. 1987. Utilization of glucose and amino acids by Bacteroides intermedius and Bacteroides gingivalis. Curr. Microbiol. 15:241-246.

18. Staley, J. T., and N. R. Krieg. 1984. Classification of procaryotic organisms: an overview, p. 1-4. In N. R. Krieg and J. G. Holt (ed.), Bergey's manual of systematic bacteriology, vol. 1. The Williams \& Wilkins Co., Baltimore.

19. Turner, A. W., A. D. Campbell, and A. T. Dick. 1935. Recent work on pleuropneumonia contagiosa bovum in North Queensland. Aust. Vet. J. 11:63-70.

20. Watabe, J., Y. Benno, and T. Mitsouka. 1983. Taxonomic study of Bacteroides oralis and related organisms and proposal of Bacteroides veroralis $\mathrm{sp}$. nov. Int. J. Syst. Bacteriol. 33:57-64. 